\title{
Upregulation of peroxiredoxin III in doxorubicin-induced cytotoxicity and the FoxO3a-dependent expression in $\mathrm{H} 9 \mathrm{c} 2$ cardiac cells
}

\author{
MI-HUA LIU ${ }^{1 *}$, YUAN ZHANG $^{2 *}$, JUN HE ${ }^{1}$, TIAN-PING TAN ${ }^{1}$, SHAO-JIAN WU ${ }^{1}$, HONG-YUN FU ${ }^{1}$, \\ YU-DAN CHEN $^{1}$, JUN LIU ${ }^{1}$, QUN-FANG LE ${ }^{1}$, HENG-JING HU ${ }^{3}$, CONG YUAN $^{4}$ and XIAO-LONG LIN ${ }^{5}$ \\ ${ }^{1}$ Department of Clinical Laboratory, Affiliated Nanhua Hospital, University of South China, Hengyang, Hunan 421001; \\ ${ }^{2}$ Department of Pathology, Hunan Mawangdui Hospital, Changsha, Hunan 410016; ${ }^{3}$ Department of Cardiology/Cardiac \\ Catheterisation Lab, Second Xiangya Hospital, Central South University, Changsha, Hunan 410011; \\ ${ }^{4}$ Department of Cardiology, The First Hospital of Changsha, Changsha, Hunan 410005; ${ }^{5}$ Department of Pathology, \\ The Third People's Hospital of Huizhou, Guangzhou Medical University, Huizhou, Guangdong 516002, P.R. China
}

Received September 18, 2014; Accepted August 3, 2015

DOI: $10.3892 /$ etm.2015.2693

\begin{abstract}
Doxorubicin (DOX) is an efficient drug used in cancer therapy; however, it produces reactive oxygen species (ROS) that induce severe cytotoxicity, limiting its clinical application. The aim of the present study was to investigate the role of peroxiredoxin III (Prx III) in DOX-induced H9c2 cell injuries. Following DOX treatment, the expression of phosphorylated-FoxO3a (p-FoxO3a) was decreased and Prx III expression was increased in $\mathrm{H} 9 \mathrm{c} 2$ cells. In order to detect whether oxidative stress was involved in the induction of Prx III expression by FoxO3a, exogenous $\mathrm{H}_{2} \mathrm{O}_{2}$ was used to induce oxidative stress in the $\mathrm{H} 9 \mathrm{c} 2$ cells. Apoptosis of $\mathrm{H} 9 \mathrm{c} 2$ cardiomyocytes was assessed using methyl thiazolyl tetrazolium assay and Hoechst staining. The levels of Prx III and p-FoxO3a were evaluated using western blot analysis. As expected, $\mathrm{H}_{2} \mathrm{O}_{2}$ was found to mimic the effect of DOX, decreasing the expression of p-FoxO3a and increasing the expression of Prx III. In addition, the study evaluated whether the transcription factor FoxO3a was essential for the expression of Prx III. Pretreatment of H9c 2 cells with $\mathrm{N}$-acetyl-L-cysteine (NAC), a scavenger of ROS, prior to exposure to DOX dramatically increased the phosphorylation of FoxO3a and led to a
\end{abstract}

Correspondence to: $\mathrm{Mr}$ Mi-Hua Liu, Department of Clinical Laboratory, Affiliated Nanhua Hospital, University of South China, 336 Dongfeng South Road, Hengyang, Hunan 421001, P.R. China

E-mail: mihualiu@163.com

Mr Xiao-Long Lin, Department of Pathology, The Third People's Hospital of Huizhou, Guangzhou Medical University, 1 Xuebei Road, Huizhou, Guangdong 516002, P.R. China

E-mail: 493814078@qq.com

*Contributed equally

Key words: doxorubicin, cardiomyocytes, peroxiredoxin III, FoxO3a marked reduction in Prx III expression in the H9c2 cells. In conclusion, the results of the current study suggest that FoxO3a mediates the expression of Prx III in DOX-induced injuries.

\section{Introduction}

Doxorubicin (DOX) is one of the most widely used anticancer drugs, due to its potent therapeutic effects on a variety of cancer types, including leukemia, lymphomas and breast cancer. However, the clinical use of DOX is limited by severe toxic side-effects on the heart, potentially resulting in congestive heart failure and dilated cardiomyopathy (1). Numerous studies have demonstrated that reactive oxygen species (ROS) production has been implicated in the cardiotoxicity of DOX, which ultimately results in endothelial dysfunction $(2,3)$ and cardiomyocyte apoptosis (4).

Transcription factors of the forkhead box, class O (FoxO) family are crucial regulators of the cellular stress response and promote the cellular antioxidant defense. FoxOs stimulate the transcription of genes coding for antioxidant proteins located in various subcellular compartments, such as in mitochondria, including superoxide dismutase-2 and peroxiredoxins $3 / 5$. In previous studies, resveratrol has been demonstrated to protect PC12 cells against high glucose-induced neurotoxicity via the PI3K/Akt/FoxO3a pathway. Various antioxidant pathways regulate or protect the cellular response to oxidative stress. Several antioxidants, including peroxiredoxins (Prxs), are components of a ubiquitous thioredoxin-dependent antioxidant defense system, which catalyzes ROS inactivation in mammalian cells (5-7). Frequently, multiple mammalian Prxs (including Prx I to VI) coexist in various intracellular locations in the same cell $(8,9)$. These act as scavengers of cellular $\mathrm{H}_{2} \mathrm{O}_{2}$ that is released following stimulation with various growth factors during apoptosis, oxidative stress or proliferation $(8,9)$. In particular, Prx III has been demonstrated to exhibit a protective role in cisplatin- and gentamicin-induced apoptosis through a mitochondria-dependent pathway (10). Previous studies reported that overexpression of Prx III protects the 
mouse myocardium from infarction (11), whereas depletion of Prx III results in increased intracellular levels of $\mathrm{H}_{2} \mathrm{O}_{2}$, sensitizing cells to apoptotic signaling (12). The forkhead box transcription factor FoxO3a is a key transcription factor for resistance to oxidative stress. Chiribau et al (13) demonstrated that Prx III expression in human cardiac fibroblasts was regulated by FoxO3a during oxidative stress. The authors also identified specific DNA-binding elements for FoxO3a in the Prx III promoter (13). The aim of the present study was to examine whether oxidative stress is able to induce Prx III expression in an injury model of DOX-induced H9c2 cells. In addition, the study investigated whether Prx III expression is regulated by FoxO3a in $\mathrm{H} 9 \mathrm{c} 2$ cells.

\section{Materials and methods}

Materials. A methyl thiazolyl tetrazolium bromide (MTT) assay, Hoechst 33258, DOX, $\mathrm{H}_{2} \mathrm{O}_{2}$ and $\mathrm{N}$-acetyl-L-cysteine (NAC) were purchased from Sigma-Aldrich (St. Louis, MO, USA). All the medium components used in cell cultures were purchased from Thermo Fisher Scientific (Waltham, MA, USA), unless otherwise stated. H9c2 cardiac myocytes were obtained from the Shanghai Cell Library of China (cells were originally from ATCC, Manassas, VA, USA).

Cell culture. H9c2 cardiac myocytes were cultured in Dulbecco's modified Eagle's medium (DMEM) supplemented with $10 \%$ fetal bovine serum (FBS), $100 \mu \mathrm{g} / \mathrm{ml}$ streptomycin and $100 \mathrm{U} / \mathrm{ml}$ penicillin (all from Gibco Life Technologies, Carlsbad, CA, USA) in a humidified $5 \% \mathrm{CO}_{2}$ atmosphere at $37^{\circ} \mathrm{C}$. $\mathrm{H} 9 \mathrm{c} 2$ cardiac myocytes were passaged every 2 days, for 5-8 passages. Subsequently, the cells were seeded at a density of $2 \times 10^{6}$ cells/dish in $100 \mathrm{~mm}$ dishes with $10 \%$ calf serum, incubated for $24 \mathrm{~h}$ and then the medium was changed to $0.5 \%$ FBS-supplemented DMEM for 24-h starvation.

MTT assay. The MTT assay is a standard method used to assess cell viability. Prior to each experiment, H9c2 cardiac myocytes (5,000 cells/well) were seeded in 96-well microtiter plates. Following incubation with NAC for $60 \mathrm{~min}$, the cells were treated with $5 \mu \mathrm{M}$ DOX and incubated for a further $24 \mathrm{~h}$. Subsequently, $10 \mu \mathrm{l}$ MTT solution was added to each well, followed by incubation for $4 \mathrm{~h}$ at $37^{\circ} \mathrm{C}$. The absorbance was then measured at $470 \mathrm{~nm}$ and the values were used to calculate the relative ratio of cell viability. Three independent experiments were performed for each experimental condition. The various experimental groups were as follows: Control group, untreated H9c2 cells incubated in 0.5\% FBS DMEM for $24 \mathrm{~h}$; DOX group, cells treated with $5 \mu \mathrm{M}$ DOX for $24 \mathrm{~h}$; $\mathrm{DOX}+\mathrm{NAC}$ group, cells treated with 1,000 $\mu \mathrm{M}$ NAC for 60 min prior to exposure to DOX; and the $\mathrm{H}_{2} \mathrm{O}_{2}$ group, cells treated with $200 \mu \mathrm{M} \mathrm{H}_{2} \mathrm{O}_{2}$ for $12 \mathrm{~h}$.

Assessment of cardiomyocyte cell apoptosis. Apoptosis was analyzed by fluorescence microscopy with the chromatin dye, Hoechst 33258. Following various treatments, the cells were fixed in ice-cold 4\% paraformaldehyde dissolved in phosphate-buffered saline (PBS) at room temperature for 20 min. Nonspecific binding was blocked using 5\% normal goat serum in $0.01 \mathrm{M}$ PBS containing $0.3 \%$ Triton $\mathrm{X}-100$.
Next, the cells were washed twice with PBS and incubated with $10 \mu \mathrm{g} / \mathrm{ml}$ Hoechst 33258 for $10 \mathrm{~min}$ at room temperature in the dark. The cells were then visualized under a fluorescence microscope (BX50-FLA; Olympus Corporation, Tokyo, Japan). Condensed, fractured or distorted nuclei were detected in apoptotic cells, whereas a normal nuclear size and uniform fluorescence were observed in viable cells. The percentage of apoptotic cells was evaluated as follows: Number of apoptotic cells/(numbers of apoptotic cells + numbers of viable cells) x 100. Percentage of cell viability was calculated using the optical density (OD), as follows: (OD treatment group/OD control group) x 100 .

Immunohistochemical staining. The cells were cultured in glass cover slips that were placed in 6-well microtiter plates for $24 \mathrm{~h}$ and then washed three times with PBS. Next, the cells were immediately fixed with ice-cold $4 \%$ paraformaldehyde solution for $15 \mathrm{~min}$, washed three times with PBS, air-dried for $5 \mathrm{~min}$ and then incubated with $0.5 \%$ Triton X-100 for $20 \mathrm{~min}$. The cover slips were saturated with $5 \%$ bovine serum albumin in PBS for $30 \mathrm{~min}$ at room temperature and then processed for immunohistochemical staining with rabbit anti-Prx III polyclonal primary antibody (ab73349; Abcam, Cambridge, MA, USA; dilution, 1:400) for $4 \mathrm{~h}$ at $37^{\circ} \mathrm{C}$. The primary antibodies were removed by washing three times in PBS and the samples were incubated for $1 \mathrm{~h}$ with goat anti-rabbit IgG horseradish peroxidase-conjugated secondary antibodies (A0208; Beyotime Institute of Biotechnology, Shanghai, China), prior to visualization with diaminobenzidine for 10-15 min. Subsequently, the cells were washed with distilled water and then counter stained with hematoxylin (Beyotime Institute of Biotechnology). Immunohistochemical micrograph was detected using a BX50-FLA fluorescence microscope. Quantification of Prx III immunostaining was performed by calculating the integral OD (IOD; positive area $\mathrm{x}$ average density) using an Image-Pro Plus system (Media Cybernetics, Inc., Bethesda, MD, USA). The percentage of Prx III positive cells was calculated as follows: (Number of Prx III positive cells/total number of cells) x 100 .

Western blot analysis. Cells were homogenized directly into cell lysis buffer (Cell Signaling Technology, Inc., Danvers, MA, USA), and a phosphatase inhibitor cocktail (Sigma-Aldrich), and the obtained cell lysates were centrifuged at $12,000 \times \mathrm{g}$ for $10 \mathrm{~min}$ at $4^{\circ} \mathrm{C}$. Protein concentration was determined using a BCA protein assay kit (Beyotime Institute of Biotechnology), following the manufacturer's instructions. The extracted proteins were mixed with 5\% sodium dodecyl sulfate (SDS)-polyacrylamide gel electrophoresis sample buffer (Beyotime Institute of Biotechnology), then boiled at $100^{\circ} \mathrm{C}$ for $7 \mathrm{~min}$ and separated by electrophoresis on a $10 \%$ SDS-polyacrylamide gel. Subsequent to electrophoresis, the proteins were transferred to polyvinylidene difluoride membranes, which were then blocked in Tris-buffered saline-Tween 20 (TBS-T; 0.1\% Tween 20) containing $5 \%$ non-fat dry milk, for $2 \mathrm{~h}$ at room temperature with rotation. After blocking, the membranes were incubated with the following antibodies: Rabbit anti-Prx III polyclonal antibody, rabbit anti-FoxO3a polyclonal antibody (12829; Cell Signaling Technologies, Inc.; dilution, 1:2,000), and 
A

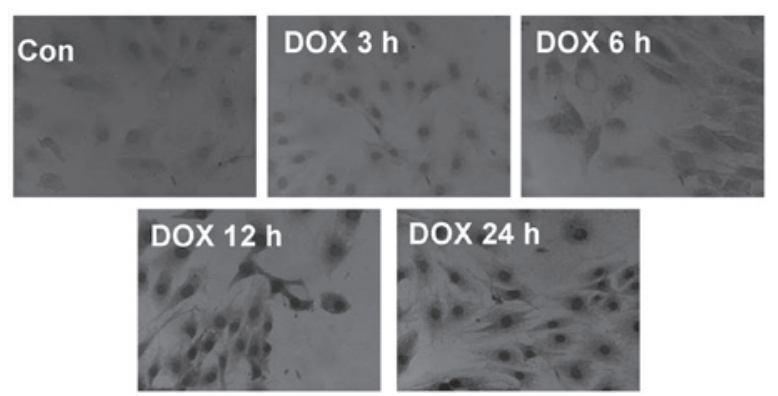

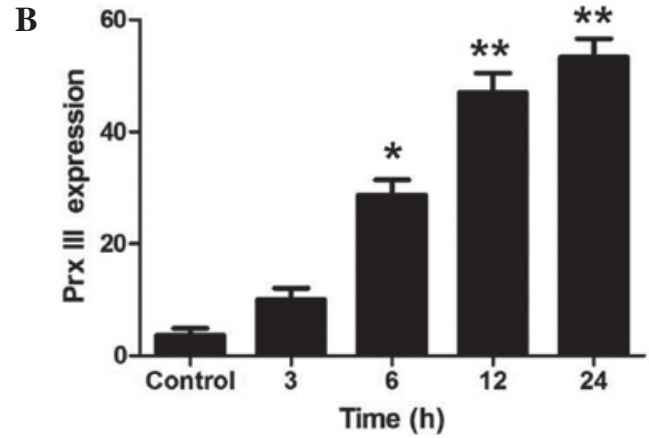

Figure 1. Prx III expression in H9c2 cells subsequent to DOX treatment for different durations. H9c2 cells were treated with $5 \mu \mathrm{M}$ DOX for 0 (control), 3,6 , 12 or $24 \mathrm{~h}$. Figure 1A: Immunohistochemical staining detected Prx III expression in H9c2 cells under a fluorescence microscope. H9c2 cells were treated with $5 \mu \mathrm{M}$ DOX for $0,3,6,12$ or $24 \mathrm{~h}$ (magnification, $\mathrm{x} 40$ ). Figure 1B: Quantitative analysis of the expression of Prx III in H9c2 cells. Data are expressed as the mean \pm standard error of mean $(n=3) . ~{ }^{*} \mathrm{P}<0.05$ and ${ }^{* *} \mathrm{P}<0.01$ vs. control group. Prx III, peroxiredoxin III; DOX, doxorubicin; Con, control.

A
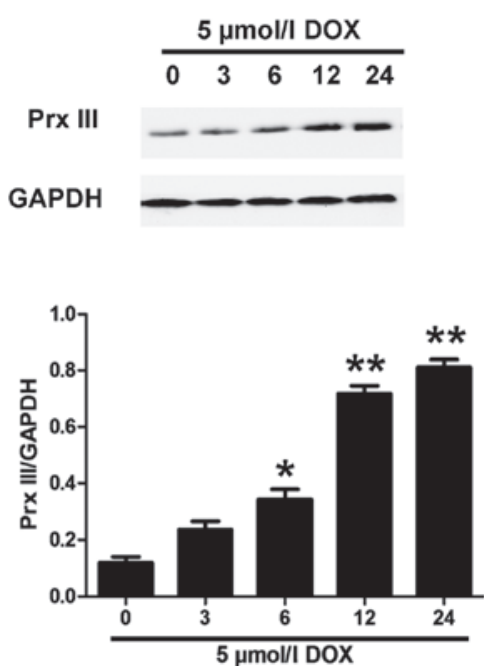

B

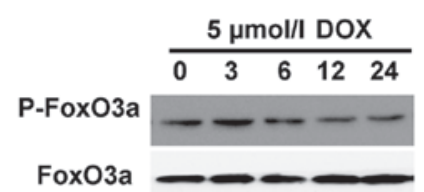

Figure 2. Effect of DOX on the expression of Prx III and phospho-FoxO3a in H9c2 cells, which were treated with $5 \mu \mathrm{M}$ DOX for $0,3,6,12$ or $24 \mathrm{~h}$. The expression of (A) Prx III and (B) p-FoxO3a was analyzed by immunoblotting (top panels). Relative levels of (A) Prx III and (B) p-FoxO3a vs. total FoxO3a in each sample were determined by western blot densitometry (bottom panels). Data are expressed as the mean \pm standard error of mean $(n=3)$. ${ }^{*} \mathrm{P}<0.05$ and ${ }^{* * *} \mathrm{P}<0.01$ vs. control group. DOX, doxorubicin; Prx III, peroxiredoxin III; p-FoxO3a, phosphorylated-FoxO3a.

rabbit anti-phosphorylated-FoxO3a (anti-p-FoxO3a; Ser 253) polyclonal antibody (13129; Cell Signaling Technologies, Inc.; dilution, 1:1,000). Subsequently, the membranes were incubated with $5 \%$ milk or bovine serum albumin overnight at $4^{\circ} \mathrm{C}$. The membranes were washed three times in TBS-T to remove the primary antibody, and incubated for $2 \mathrm{~h}$ with the appropriate horseradish peroxidase-conjugated secondary antibodies. Following washing three times in TBS-T, the antigen-antibody bands were detected using an enhanced chemiluminescence reagent kit (Beyotime Institute of Biotechnology) and quantified using Quantity One software (Bio-Rad Laboratories, Inc., Hercules, CA, USA). The data of the immunoblots of p-FoxO3a were represented as a ratio of the phosphorylated forms to their total forms.

Statistical analysis. Results are presented as the mean \pm standard error of mean. Statistical analysis was performed using Student's t-test or analysis of variance with SPSS 13.0 software (SPSS, Inc., Chicago, IL, USA). In all cases, a value of $\mathrm{P}<0.05$ was accepted as indicating a statistically significant difference.

\section{Results}

DOX increases Prx III expression in a time-dependent manner. In order to elucidate whether PrX III was associated with DOX-induced injuries in H9c2 cells, the expression of Prx III was observed. H9c2 cells were treated with $5 \mu \mathrm{M}$ DOX for the indicated times (0, 3, 6, 12 and $24 \mathrm{~h})$. Immunohistochemical staining (Fig. 1) and western blot analysis (Fig. 2A) revealed that Prx III protein expression was significantly upregulated in $\mathrm{H} 9 \mathrm{c} 2$ cells after $6 \mathrm{~h}$ of incubation with $5 \mu \mathrm{M}$ DOX. The expression of Prx III increased as the incubation time was prolonged, with the strongest effect observed in the $24 \mathrm{~h}$ group.

DOX treatment decreases $p$-FoxO3a expression in $\mathrm{H} 9 \mathrm{c} 2$ cells. A previous study reported that FoxO3a mediated Prx III expression (13). The aforementioned results (Figs. 1 and 2A) demonstrated that DOX treatment induced Prx III expression in H9c2 cells; subsequently, we attempted to investigate whether FoxO3a regulates the expression of Prx III in DOX-induced $\mathrm{H} 9 \mathrm{c} 2$ cell injury. Therefore, the expression of $\mathrm{p}-\mathrm{FoxO} 3 \mathrm{a}$ was evaluated in the present study. H9c2 cells were treated with 
A
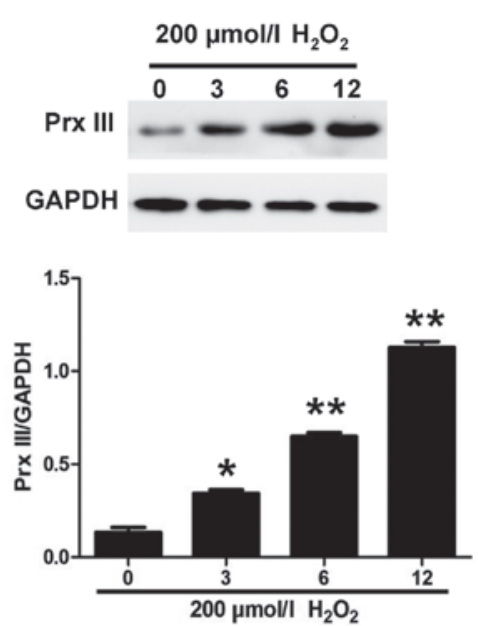

B
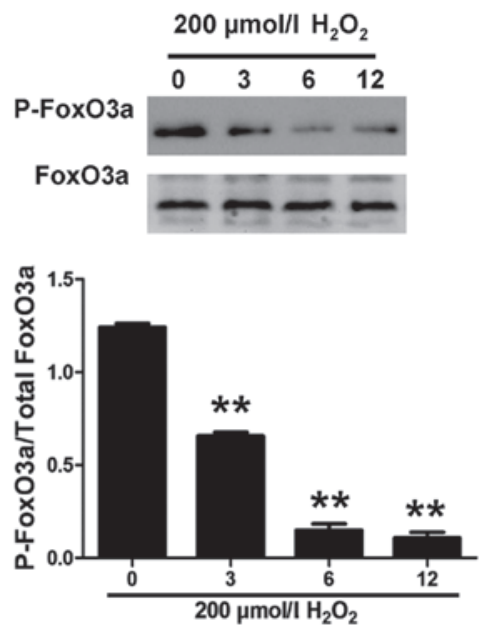

Figure 3. Role of oxidative stress on DOX-induced expression of Prx III and p-FoxO3a in H9c2 cells, which were treated with $\mathrm{H}_{2} \mathrm{O}_{2}$ at a concentration of $200 \mu \mathrm{mol} / 1$ for 0,3,6 or $12 \mathrm{~h}$. The expression of (A) Prx III and (B) p-FoxO3a was analyzed by immunoblotting (top panels). Relative levels of (A) Prx III and (B) p-FoxO3a vs. total FoxO3a in each sample were determined by western blot densitometry (bottom panels). Data are expressed as the mean \pm standard error of mean $(\mathrm{n}=3) .{ }^{*} \mathrm{P}<0.05$ and ${ }^{* * *} \mathrm{P}<0.01$ vs. control group. DOX, doxorubicin; Prx III, peroxiredoxin III; p-FoxO3a, phosphorylated-FoxO3a.

A

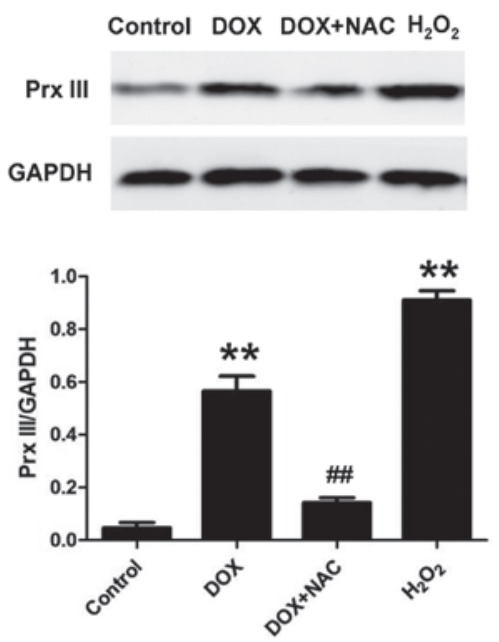

B
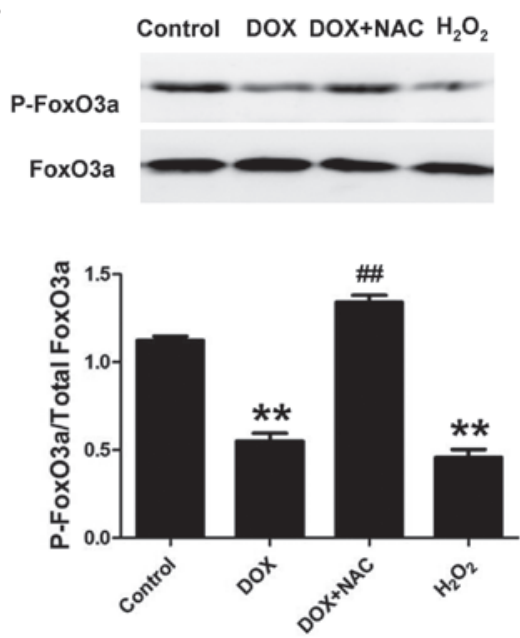

Figure 4. Effect of an antioxidant on DOX-induced expression of PrX III and p-FoxO3a in H9c2 cells. The cells were treated with $5 \mu \mathrm{M}$ DOX for $24 \mathrm{~h}$, and treated or pretreated with 1,000 $\mu \mathrm{M}$ NAC for $60 \mathrm{~min}$. The expression of (A) Prx III and (B) p-FoxO3a was analyzed by immunoblotting (top panels). Relative levels of (A) Prx III and (B) p-FoxO3a vs. total FoxO3a in each sample were determined by western blot densitometry (bottom panels). Data are expressed as the mean \pm standard error of mean $(\mathrm{n}=3) .{ }^{* *} \mathrm{P}<0.01$, vs. control group; ${ }^{\# \#} \mathrm{P}<0.01$, vs. DOX-treated group. DOX, doxorubicin; Prx III, peroxiredoxin III; $\mathrm{p}$-FoxO3a, phosphorylated-FoxO3a; NAC, N-acetyl-L-cysteine.

$5 \mu \mathrm{M}$ DOX for the indicated times $(0,3,6,12$ and $24 \mathrm{~h})$. As expected, DOX treatment significantly decreased the expression of p-FoxO3a in a time-dependent manner (Fig. 2B). However, $5 \mu \mathrm{M}$ DOX alone did not induce significant changes in the expression of total FoxO3a. These findings suggest that the expression of non-phosphorylated FoxO3a was significantly increased following DOX stimulation, which induced Prx III expression in H9c2 cells (Fig. 2A).

FoxO3a is required for the expression of Prx III in $\mathrm{H} 9 \mathrm{c} 2$ cells. In order to determine whether oxidative stress was involved in the induction of Prx III expression by FoxO3a, exogenous $\mathrm{H}_{2} \mathrm{O}_{2}$ was used to induce oxidative stress in the H9c2 cells (Fig. 3). As shown in Fig. 3B, the exposure of $\mathrm{H} 9 \mathrm{c} 2$ cells to $200 \mu \mathrm{mol} / 1$ $\mathrm{H}_{2} \mathrm{O}_{2}$ for the indicated times $(0,3,6$ and $12 \mathrm{~h})$ caused a significant downregulation of p-FoxO3a expression in $\mathrm{H} 9 \mathrm{c} 2$ cells. In addition, the expression of Prx III following $\mathrm{H}_{2} \mathrm{O}_{2}$ treatment was examined and was found to increase (Fig. 3A). These data suggested that oxidative stress induces Prx III expression in a FoxO3a-dependent manner in $\mathrm{H} 9 \mathrm{c} 2$ cells.

Oxidative stress on the expression of Prx III and p-FoxO3a in $\mathrm{H} 9 \mathrm{c} 2$ cells. To further confirm whether the DOX-induced expression of Prx III is associated with oxidative stress, H9c2 cells were pretreated with 1,000 $\mu \mathrm{M} \mathrm{NAC} \mathrm{(a} \mathrm{ROS} \mathrm{scavenger)} \mathrm{for}$ $60 \mathrm{~min}$ prior to exposure to $5 \mu \mathrm{M}$ DOX for $24 \mathrm{~h}$. As shown in Fig. 4, the pretreatment of cells with NAC for 60 min markedly increased the expression of p-FoxO3a and depressed the expression of Prx III. However, treatment with $1,000 \mu \mathrm{MNAC}$ alone did not significantly alter the expression of total FoxO3a. The results revealed that oxidative stress contributed to the DOX-induced Prx III expression in a FoxO3a-dependent manner. 


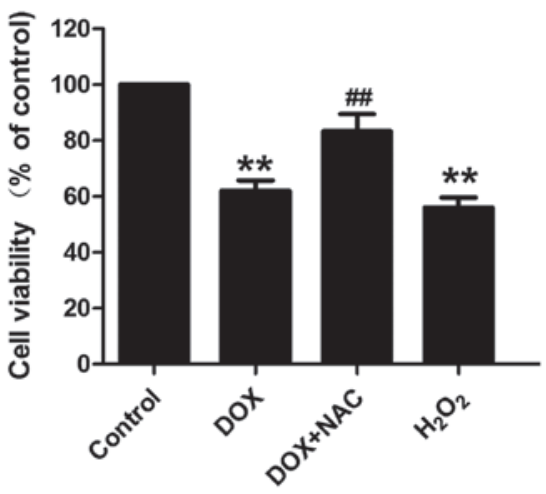

Figure 5. Cell viability was measured using an MTT assay, in order to determine the effect of antioxidant on DOX-induced cytotoxicity in H9c2 cells. Data are expressed as the mean \pm standard error of mean $(n=3) .{ }^{* *} \mathrm{P}<0.01$, vs. control group; ${ }^{\# \#} \mathrm{P}<0.01$, vs. DOX-treated group. DOX, doxorubicin; NAC, $\mathrm{N}$-acetyl-L-cysteine; control group, untreated H9c2 cells; DOX group, cells treated with $5 \mu \mathrm{M}$ DOX for $24 \mathrm{~h}$; DOX + NAC group, cells treated with $1,000 \mu \mathrm{M}$ NAC for 60 min prior to exposure to DOX; $\mathrm{H}_{2} \mathrm{O}_{2}$ group, cells treated with $200 \mu \mathrm{M} \mathrm{H}_{2} \mathrm{O}_{2}$ for $12 \mathrm{~h}$.
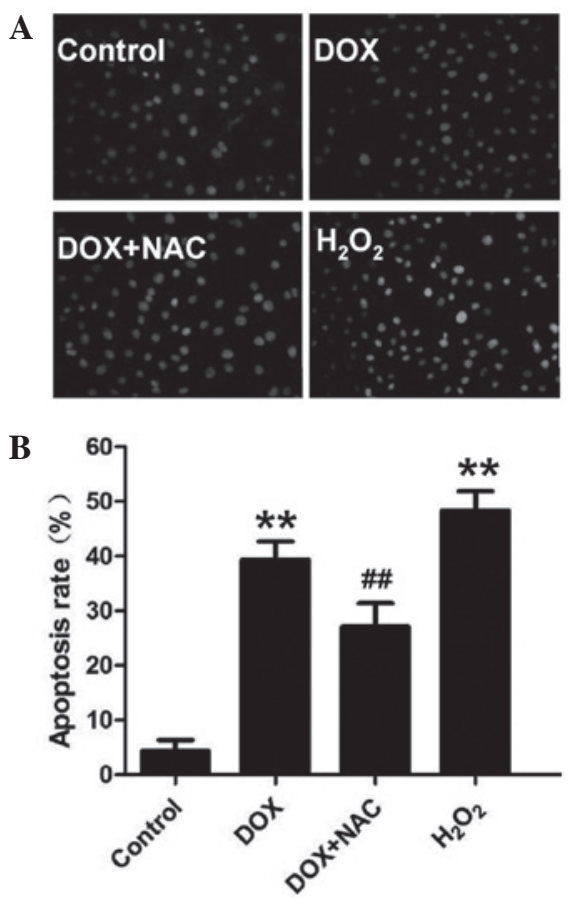

Figure 6. Effect of antioxidant on DOX-induced apoptosis in H9c2 cells. (A) Hoechst 33258 nuclear staining followed by fluorescence imaging, performed to observe cell apoptosis (magnification, x40). (B) The apoptotic rate was analyzed with a cell counter and Image J 1.41o software. Data are expressed as the mean \pm standard error of mean $(n=3) .{ }^{* *} \mathrm{P}<0.01$, vs. control group; ${ }^{\# \#} \mathrm{P}<0.01$, vs. DOX-treated group. DOX, doxorubicin; NAC, $\mathrm{N}$-acetyl-L-cysteine; control group, untreated H9c2 cells; DOX group, cells treated with $5 \mu \mathrm{M}$ DOX for $24 \mathrm{~h}$; DOX + NAC group, cells treated with $1,000 \mu \mathrm{M}$ NAC for $60 \mathrm{~min}$ prior to exposure to DOX; $\mathrm{H}_{2} \mathrm{O}_{2}$ group, cells treated with $200 \mu \mathrm{M} \mathrm{H}_{2} \mathrm{O}_{2}$ for $12 \mathrm{~h}$.

Oxidative stress mediated DOX-induced cytotoxicity and apoptosis in $H 9 c 2$ cells. As shown in Fig. 5, exposure of $\mathrm{H} 9 \mathrm{c} 2$ cells to DOX at $5 \mu \mathrm{M}$ for $24 \mathrm{~h}$ induced marked cytotoxicity, leading to a decrease in cell viability. As shown in Fig. 6, H9c2 cells treated with $5 \mu \mathrm{M}$ DOX for $24 \mathrm{~h}$ exhibited typical characteristics of apoptosis, including condensation of chromatin, shrinkage of nuclei and apoptotic bodies. To elucidate whether oxidative stress involved in DOX-induced cytotoxicity and apoptosis, $\mathrm{H} 9 \mathrm{c} 2$ cells were preconditioned with a well-known ROS scavenger, NAC $(1,000 \mu \mathrm{M})$ prior to DOX treatment. The results showed that pretreatment of cells with NAC significantly attenuated DOX-induced cytotoxicity (Fig. 5) and apoptosis (Fig. 6). In addition, the exogenous ROS $\mathrm{H}_{2} \mathrm{O}_{2}$ induced marked cytotoxicity and apoptosis, exhibiting a similar function to that of DOX. These results indicate that oxidative stress contributes to DOX-induced cytotoxicity and apoptosis in $\mathrm{H} 9 \mathrm{c} 2$ cells.

\section{Discussion}

Doxorubicin (DOX) is one of the most widely used and efficient antitumor drugs. However, its clinical use is limited by its severe cumulative dose-associated cardiotoxicity (14). Numerous studies have demonstrated that ROS generation due to the catalytic quinone moiety of DOX is the major molecular mechanism involved in DOX-induced cardiac toxicity, inducing cardiomyocyte apoptosis (15).

Prx III, a member of the Prx family, is a mitochondrial antioxidant protein that is capable of catalyzing $\mathrm{H}_{2} \mathrm{O}_{2}$ reduction (16). Prx III overexpression has been reported to protect neurons against cell death induced by oxidative stress (17). Due to these characteristics, Prx III is an important candidate for the treatment against left ventricular failure after myocardial infarction, during which an increased production of ROS has been observed within the mitochondria (11). Although various studies have previously demonstrated the beneficial effects of antioxidants on heart failure (18), no previous studies have specifically investigated the protective role of Prx III in DOX-induced cytotoxicity, to the best of our knowledge. In the present study, Prx III was found to be significantly increased in an injury model established by DOX-treatment in H9c2 cells. Similar to the findings of the current study, an increase in Prx III expression was previously reported by Xi et al (19), and nitrate treatment was found to completely restore the expression of Prx III.

FoxO3a has been recently shown to be a key transcription factor involved in resistance to oxidative stress (20). When cells are exposed to oxidative stress, FoxO3a translocates to the nucleus and activates transcription by specifically binding to the consensus sequence TTGTTTAC in the promoters of target genes (21). A previous study revealed that FoxO3a increased the resistance to oxidative stress by upregulating the expression of Prx III in human cardiac fibroblasts (13). Therefore, the present study evaluated the regulation of FoxO3a in Prx III expression. Treatment of H9c2 cells with DOX was found to significantly inhibit the expression of $\mathrm{p}$-FoxO3a in a time-dependent manner. Following the downregulation of p-FoxO3a expression, Prx III expression was significantly higher in H9c2 cells treated with DOX, suggesting an indispensable role of Prx III in the protection against oxidative stress.

Increasing evidence has suggested a major role for ROS in the pathogenesis of cardiac failure (22). Furthermore, antioxidants have been demonstrated to exert protective and beneficial effects against heart failure (11). DOX induces cardiomyocyte insult mainly by oxidative stress. In the current study, in order to determine whether oxidative stress was 
involved in the induction of Prx III expression by FoxO3a, exogenous $\mathrm{H}_{2} \mathrm{O}_{2}$ was used to induce oxidative stress in $\mathrm{H} 9 \mathrm{c} 2$ cells. As evidenced in the present study, $\mathrm{H}_{2} \mathrm{O}_{2}$ mimicked the effect of DOX, resulting in a decrease in p-FoxO3a expression and an increase in Prx III expression, after H9c2 cells were treated with $\mathrm{H}_{2} \mathrm{O}_{2}$. The upregulation of Prx III in H9c2 cells would help cells to remove excessive ROS, providing a favorable microenvironment for cell proliferation and enhancing cardiomyocyte survival. These findings suggest that ROS may function as an important mediator in the induction of Prx III expression by FoxO3a following DOX treatment in $\mathrm{H} 9 \mathrm{c} 2$ cells.

To further confirm that FoxO3a plays an essential role in the mediation of Prx III expression in a DOX-treated H9c2 cell injury model, H9c2 cells were treated with $1,000 \mu \mathrm{mol} / 1 \mathrm{NAC}$ (a ROS scavenger) for $60 \mathrm{~min}$ prior to exposure to DOX. The results indicated that NAC significantly increased p-FoxO3a expression, resulting in the suppression of Prx III expression. The results also revealed that the antioxidant effect of NAC suppressed the DOX-induced Prx III expression, suggesting that the expression of Prx III was dependent on FoxO3a. These findings suggest that FoxO3a regulated the expression of Prx III and protected against oxidative stress by increasing Prx III expression.

Peroxiredoxin (Prx) III is an antioxidant enzyme that controls cytokine-induced peroxide levels. In a previous study, Jeong et al (9) suggested that FoxO3a mediates the neuronal levels of the expression of Prx III and the levels of expression of Mn-SOD in vivo. The present results are consistent with these previous findings, demonstrating for the first time that mitochondrial Prx III was upregulated in DOX-treated H9c2 rat embryonic cardiomyocytes. Jeong et al (9) and the present study demonstrated that oxidative stress altered the expression of Prx III, suggesting that Prx III may be used as a novel therapeutic targeting DOX-induced cytotoxicity.

In conclusion, the present study demonstrated for the first time that mitochondrial Prx III was upregulated in DOX-treated H9c2 rat embryonic cardiomyocytes. The study provided evidence that Prx III is an important regulator of intracellular ROS, suggesting that upregulation of Prx III expression may be used as a novel therapeutic strategy to protect against DOX-induced cardiotoxicity.

\section{Acknowledgements}

This study was supported by grants from the Medical Scientific Research Funds of Guangdong province (no. A2014810) and the Graduate Student Research Innovation Project of Hunan province (no. CX2013B397).

\section{References}

1. Magnano LC, Martínez Cibrian N, Andrade González X and Bosch X: Cardiac complications of chemotherapy: Role of prevention. Curr Treat Options Cardiovasc Med 16: 312, 2014.

2. Jang WJ, Choi DY and Jeon IS: Vascular endothelial dysfunction after anthracyclines treatment in children with acute lymphoblastic leukemia. Korean J Pediatr 56: 130-134, 2013.
3. Truong J, Yan AT, Cramarossa G and Chan KK: Chemotherapy-induced cardiotoxicity: detection, prevention and management. Can J Cardiol 30: 869-878, 2014.

4. Spagnuolo RD, Recalcati S, Tacchini L and Cairo G: Role of hypoxia-inducible factors in the dexrazoxane-mediated protection of cardiomyocytes from doxorubicin-induced toxicity. Br J Pharmacol 163: 299-312, 2011.

5. Klotz LO, Sánchez-Ramos C, Prieto-Arroyo I, Urbánek P, Steinbrenner $\mathrm{H}$ and Monsalve $\mathrm{M}$ : Redox regulation of FoxO transcription factors. Redox Biol 6: 51-72, 2015.

6. Liu MH, Yuan C, He J, Tan TP, Wu SJ, Fu HY, Liu J, Yu S, Chen YD, Le QF, et al: Resveratrol protects PC12 cells from high Glucose-induced neurotoxicity via PI3K/Akt/FoxO3a pathway. Cell Mol Neurobiol 35: 513-522, 2015.

7. Fiuza B, Subelzú N, Calcerrada P, Straliotto MR, Piacenza L, Cassina A, Rocha JB, Radi R, de Bem AF and Peluffo G: Impact of SIN-1-derived peroxynitrite flux on endothelial cell redox homeostasis and bioenergetics: Protective role of diphenyl diselenide via induction of peroxiredoxins. Free Radic Res 49: 122-132, 2015

8. Poynton RA and Hampton MB: Peroxiredoxins as biomarkers of oxidative stress. Biochim Biophys Acta 1840: 906-912, 2014.

9. Jeong HJ, Jeong HW, Song SS, Kang JW, Seo JH, Lee YH, Lee KS and Kim DW: Upregulation of peroxiredeoxin III in the hippocampus of acute immobilization stress model rats and the Foxo3a-dependent expression in PC12 cells. Cell Mol Neurobiol 31: 1041-1046, 2011.

10. Chae HZ, Kim HJ, Kang SW and Rhee SG: Characterization of three isoforms of mammalian peroxiredoxin that reduce peroxides in the presence of thioredoxin. Diabetes Res Clin Pract 45: 101-112, 1999.

11. Matsushima S, Ide T, Yamato M, Matsusaka H, Hattori F, Ikeuchi M, Kubota T, Sunagawa K, Hasegawa Y, Kurihara T, et al: Overexpression of mitochondrial peroxiredoxin-3 prevents left ventricular remodeling and failure after myocardial infarction in mice. Circulation 113: 1779-1786, 2006.

12. Chang TS, Cho CS, Park S, Yu S, Kang SW and Rhee SG: Peroxiredoxin III, a mitochondrion-specific peroxidase, regulates apoptotic signaling by mitochondria. J Biol Chem 279: 41975-41984, 2004.

13. Chiribau CB, Cheng L, Cucoranu IC, Yu YS, Clempus RE and Sorescu D: FOXO3A regulates peroxiredoxin III expression in human cardiac fibroblasts. J Biol Chem 283: 8211-8217, 2008.

14. Lipshultz SE, Karnik R, Sambatakos P, Franco VI, Ross SW and Miller TL: Anthracycline-related cardiotoxicity in childhood cancer survivors. Curr Opin Cardiol 29: 103-112, 2014.

15. Salazar-Mendiguchía J, González-Costello J, Roca J, Ariza-Sole A, Manito N and Cequier A: Anthracycline-mediated cardiomyopathy: Basic molecular knowledge for the cardiologist. Arch Cardiol Mex 84: 218-223, 2014.

16. Song IS, Kim HK, Jeong SH, Lee SR, Kim N, Rhee BD, Ko KS and Han J: Mitochondrial peroxiredoxin III is a potential target for cancer therapy. Int J Mol Sci 12: 7163-7185, 2011.

17. Hattori F, Murayama N, Noshita T and Oikawa S: Mitochondrial peroxiredoxin-3 protects hippocampal neurons from excitotoxic injury in vivo. J Neurochem 86: 860-868, 2003.

18. Betge S, Lutz K, Roskos M and Figulla HR: Oral treatment with probucol in a pharmacological dose has no beneficial effects on mortality in chronic ischemic heart failure after large myocardial infarction in rats. Eur J Pharmacol 558: 119-127, 2007.

19. Xi L, Zhu SG, Hobbs DC and Kukreja RC: Identification of protein targets underlying dietary nitrate-induced protection against doxorubicin cardiotoxicity. J Cell Mol Med 15: 2512-2524, 2011.

20. Storz P: Forkhead homeobox type O transcription factors in the responses to oxidative stress. Antioxid Redox Signal 14: 593-605, 2011.

21. Pierrou S, Hellqvist M, Samuelsson L, Enerbäck S and Carlsson P: Cloning and characterization of seven human forkhead proteins: binding site specificity and DNA bending. EMBO J 13: 5002-5012, 1994.

22. Schwarzer M, Osterholt M, Lunkenbein A, Schrepper A, Amorim P and Doenst T: Mitochondrial reactive oxygen species production and respiratory complex activity in rats with pressure overload-induced heart failure. J Physiol 592: 3767-3782, 2014. 